\title{
O conhecimento de métodos contraceptivos entre universitárias de enfermagem
}

\author{
Valdecila Luiza Lacerda da Silva ${ }^{11}$ \\ Maria de Lourdes Pereira ${ }^{2}$ \\ Susana da Silva Martins ${ }^{3}$ \\ Geisa Sant'Ana ${ }^{4}$
}

\section{Resumo}

O comportamento sexual e reprodutivo do ser humano relaciona-se ao nível de escolaridade, pois aqueles que se dedicam mais aos estudos fazem uso dos métodos contraceptivos e evitam a gravidez indesejada e/ou as DSTs. Esta pesquisa de abordagem quantitativa foi possível a partir de um questionário autoaplicado sobre o estudo de corte transversal. A amostra foi composta por 100 universitárias com idade média de 26 anos. A maioria declarou ter conhecimento de algum método contraceptivo, mas quanto ao uso, distorções foram identificadas. Verificou-se, que o conhecimento afirmado entre as universitárias parece não estabelecer relação coerente ao autocuidado necessário para um comportamento contraceptivo planejado e seguro. Constatou-se, portanto, que melhores estratégias norteiem essas universitárias de Enfermagem que irão compor o quadro de futuros profissionais de saúde.

Palavras-chave: Escolaridade. Comportamento. Contracepção.

\section{Introdução}

O perfil sexual e contraceptivo de jovens brasileiros deve ser entendido mediante sua inserção nas Universidades, uma vez que estudos apontam que aqueles que alcançam o mais alto nível de escolaridade mostram-se, em sua maior parte, como um grupo que

${ }^{1}$ Graduanda em Enfermagem pela Faculdade LS. E-mail: valdecilaenfermagem@yahoo. com.br.

2 Graduanda em Enfermagem pela Faculdade LS. E-mail: marialpc19@yahoo.com.br.

${ }^{3}$ Graduanda em Enfermagem pela Faculdade LS. E-mail: indiaenf@hotmail.com.

${ }^{4}$ Enfermeira, Mestre pela Faculdade de Ciências da Saúde (FS) da Universidade de Brasília (UnB), Professora da Faculdade LS. E-mail: geisa.santana0@gmail.com 
adia as uniões e as gestações para um futuro mais distante, quando houver certo sucesso profissional aliado à emancipação da família de origem (PIROTTA; SCHOR, 2004).

O impacto que a alta escolarização tem sobre inúmeros aspectos do processo saúde-doença de indivíduos e grupos sociais, incluindo a sua saúde sexual e reprodutiva, é fato a ser evidenciado. Sabe-se que o maior número de anos de estudo estaria relacionado com o processo de a mulher postergar as uniões conjugais, fazer uso frequente de métodos anticoncepcionais de alta eficácia e apresentar tendência a enfrentar menos gestações não planejadas, além de ter maior acesso material aos métodos mais modernos, não em serviços públicos, mas em clínicas particulares e farmácias (BASTOS et al., 2008).

O uso adequado dos métodos anticoncepcionais impede a ocorrência de gestações não desejadas, além de proporcionar a prevenção de doenças sexualmente transmissíveis e a AIDS (SANTOS et al., 2009).

Importante ressaltar que, desde os anos sessenta, as mulheres brasileiras vinham processando a ruptura com o clássico e exclusivo papel social que lhes era atribuído pela maternidade, introduzindo-se no mercado de trabalho e ampliando suas aspirações de cidadania (COSTA et al., 2006).

O Ministério da Saúde afirma que os profissionais devem estar preparados para lidar com mitos, preconceitos e percepções errôneas que os indivíduos acumulam com relação aos Métodos Anticoncepcionais (MAC), sexualidade, saúde reprodutiva, acompanhamento dos filhos, dentre outros (MOURA, 2005).

Observar o comportamento contraceptivo de universitárias de Enfermagem é antecipar como esses futuros profissionais estão hoje resolvendo suas questões sexuais e contraceptivas, desenvolvendo um olhar de possibilidades na assistência que prestaram às jovens mulheres.

A prevenção temporária da fertilidade é a contracepção. A eficácia da contracepção depende da motivação, que é resultado de educação, cultura, religião e situação pessoal (NETTINA, 2007).

$\mathrm{O}$ assunto discutido aqui foi um tema de consenso entre as alunas. Partiu-se da ideia de esclarecer como as alunas de Enfermagem do Distrito Federal estariam vivenciando as questões relacionadas à anticoncepção. 
Em razão disso, foi proposto um estudo do perfil sexual das universitárias, escolhendo-se as estudantes da saúde (Enfermagem) do DF, com o objetivo de investigar as condutas e práticas voltadas para a contracepção; identificar os métodos mais utilizados e averiguar a regularidade do uso.

\section{Metodologia}

A presente pesquisa, com abordagem quantitativa, tem como metodologia um estudo de corte transversal. A amostra foi composta por 100 acadêmicas de Enfermagem de cinco Faculdades de Enfermagem do Distrito Federal. Foram considerados sujeitos das pesquisas, aquelas que aceitaram participar e assinaram o Termo de Consentimento Livre e Esclarecido, no qual ficou assegurada a preservação de identidade e de informações colhidas no questionário.

Para coleta dos dados, foi distribuído um questionário autoaplicado, composto por quinze perguntas objetivas, o qual aborda dados demográficos (idade), sociais (renda, estado civil, ocupação, moradia, tipo de assistência médica), práticas e conhecimento sobre contracepção.

Para análise e interpretação dos dados, foi utilizado o tratamento estatístico por meio do programa Epi Info versão 3.4.1/2007 e Excel, em que foram exploradas e organizadas tabelas e discutiram-se os resultados com o auxílio de literatura pertinente.

O projeto de pesquisa foi aprovado pelo Comitê de Ética e pesquisa do UniCEUB, após apreciação e verificação de atendimento às normas exigidas.

\section{Resultados}

Os questionários foram aplicados em novembro de 2009 e todas aquelas que aceitaram participar espontaneamente da pesquisa assinaram o Termo de Consentimento Livre e Esclarecido (TCLE). Os questionários foram aplicados somente em Instituições particulares de nível superior do DF.

$\mathrm{Na}$ Tabela 1, a faixa etária da maioria das participantes é de 26 anos $(\mathrm{DP}=8)$, variando entre 18 e 60 anos; 70\% na faixa dos 20 aos 29 anos. A maioria das estudan- 


\section{2

tes (35\%) cursava o $2^{\circ}$ semestre do curso de Enfermagem, $33 \%$ pertenciam à classe $\mathrm{E}$ (IBGE). Quanto ao tipo de moradia, $74 \%$ alegaram possuir casa própria. Identificouse também que, quanto à situação ocupacional, 58\% trabalham e $42 \%$ não.

Tabela 1 - Características sócio-demográficas de Universitárias de Enfermagem do Distrito Federal, Brasil.

\begin{tabular}{|c|c|c|}
\hline $\begin{array}{l}\text { Variáveis selecionadas - Total da amostra (100) } \\
\text { Idade (anos) }\end{array}$ & No & $\%$ \\
\hline Média & 26 & ---- \\
\hline Desvio padrão & 8 & $-\cdots$ \\
\hline \multicolumn{3}{|l|}{ Distribuição (faixa etária) } \\
\hline 15 a19 anos & 11 & $11 \%$ \\
\hline 20 a 29 anos & 70 & $70 \%$ \\
\hline 30 a 39 anos & 14 & $14 \%$ \\
\hline 40 a 49 anos & 2 & $2 \%$ \\
\hline 50 a 59 anos & 2 & $2 \%$ \\
\hline 60 a 69 anos & 1 & $1 \%$ \\
\hline \multicolumn{3}{|l|}{ Escolaridade } \\
\hline $1^{\circ}$ Semestre & 2 & $2 \%$ \\
\hline $2^{\circ}$ Semestre & 35 & $35 \%$ \\
\hline $3^{\circ}$ Semestre & 30 & $30 \%$ \\
\hline $4^{\circ}$ Semestre & 8 & $8 \%$ \\
\hline $5^{\circ}$ Semestre & 9 & $9 \%$ \\
\hline $6^{\circ}$ Semestre & 5 & $5 \%$ \\
\hline $7^{\circ}$ Semestre & 1 & $1 \%$ \\
\hline $8^{\circ}$ Semestre & 10 & $10 \%$ \\
\hline \multicolumn{3}{|l|}{ Renda Mensal } \\
\hline Classe E & 33 & $33 \%$ \\
\hline Classe D & 22 & $22 \%$ \\
\hline Classe C & 4 & $4 \%$ \\
\hline Ignorado & 41 & $41 \%$ \\
\hline \multicolumn{3}{|l|}{ Tipo de Moradia } \\
\hline Alugada & 26 & $26 \%$ \\
\hline Casa própria & 74 & $74 \%$ \\
\hline \multicolumn{3}{|l|}{ Estado Civil } \\
\hline Solteira & 75 & $75 \%$ \\
\hline Casada & 24 & $24 \%$ \\
\hline Divorciada & 1 & $1 \%$ \\
\hline
\end{tabular}


Tabela 2 - Características da vida sexual de Universitárias de Enfermagem do DF, Brasil.

\begin{tabular}{|c|c|c|}
\hline Variáveis selecionadas - Total da amostra (100) & No & $\%$ \\
\hline \multicolumn{3}{|l|}{ Número de filhos vivos } \\
\hline Nenhum & 71 & $71 \%$ \\
\hline 1 & 11 & $11 \%$ \\
\hline 2 & 10 & $10 \%$ \\
\hline 3 ou mais & 5 & $5 \%$ \\
\hline Ignorado & 3 & $3 \%$ \\
\hline \multicolumn{3}{|l|}{ Idade da iniciação sexual } \\
\hline 10 a 15 anos & 18 & $18 \%$ \\
\hline 16 a 21 anos & 60 & $60 \%$ \\
\hline 22 a 27 anos & 11 & $11 \%$ \\
\hline$>28$ anos & 0 & $0 \%$ \\
\hline Não teve relação sexual (Virgem) & 11 & $11 \%$ \\
\hline \multicolumn{3}{|l|}{ Uso de método contraceptivo na $1^{\circ}$ relação } \\
\hline Sim & 58 & $58 \%$ \\
\hline Não & 31 & $31 \%$ \\
\hline Ignorado & 11 & $11 \%$ \\
\hline \multicolumn{3}{|l|}{ Tem vida sexual ativa } \\
\hline Sim & 77 & $77 \%$ \\
\hline Não & 12 & $12 \%$ \\
\hline Ignorado & 11 & $11 \%$ \\
\hline \multicolumn{3}{|l|}{$\begin{array}{l}\text { Número de parceiros/ desde que iniciou sua vida } \\
\text { sexual }\end{array}$} \\
\hline 1 & 32 & $32 \%$ \\
\hline 2 a 5 & 41 & $41 \%$ \\
\hline 6 a 9 & 12 & $12 \%$ \\
\hline $10 \mathrm{ou}+$ & 4 & $4 \%$ \\
\hline Ignorado & 11 & $11 \%$ \\
\hline \multicolumn{3}{|l|}{ Já teve alguma DST } \\
\hline Nenhum & 83 & $83 \%$ \\
\hline $\mathrm{HPV}$ & 2 & $2 \%$ \\
\hline Candidiase & 3 & $3 \%$ \\
\hline Gonorréia & 1 & $1 \%$ \\
\hline Sífilis & 1 & $1 \%$ \\
\hline Triconoma Vaginal & 1 & $1 \%$ \\
\hline
\end{tabular}

Na Tabela 2, referente às características da vida sexual, a média de idade de iniciação sexual foi na faixa etária de 16 aos 21 anos (60\%), 71\% delas não têm 
filhos, $77 \%$ relatam ter vida sexual ativa e $41 \%$ já tiveram de dois a cinco parceiros e $32 \%$ das universitárias afirmaram ter relação somente com um parceiro.

Do total, $58 \%$ das estudantes afirmaram ter utilizado algum método contraceptivo na primeira relação sexual. Questionadas sobre antecedentes de doenças sexualmente transmissíveis (DSTs), 83\% negaram histórico das doenças.

Na Tabela 3, identificamos a camisinha masculina com maior destaque de conhecimento (C) (99\%), seguida pela pílula (91\%), injetáveis (89\%), camisinha feminina (87\%), DIU (78\%), coito interrompido (74\%), ao passo que a utilização (U) da camisinha masculina é vista em $80 \%, 68 \%$ pílula, $35 \%$ coito interrompido. A (U) da camisinha feminina, apesar de conhecida, ainda é pouco utilizada (24\%).

Tabela 3 - Conhecimento (C) sobre os métodos contraceptivos e sua utilização (U) pelas Universitárias de Enfermagem

\begin{tabular}{|l|c|c|c|c|}
\hline \multicolumn{1}{|c|}{ Total da amostra (100) } & (C) No & $\mathbf{\%}$ & (U) No & \% \\
\hline Camisinha masculina & 99 & $99 \%$ & 80 & $80 \%$ \\
\hline Camisinha feminina & 87 & $87 \%$ & 24 & $24 \%$ \\
\hline Coito interrompido & 74 & $74 \%$ & 35 & $35 \%$ \\
\hline Ogino-Knaus “tabelinha" & 62 & $62 \%$ & 9 & $9 \%$ \\
\hline Antc Injetável & 89 & $89 \%$ & 25 & $25 \%$ \\
\hline Pílula & 91 & $91 \%$ & 68 & $68 \%$ \\
\hline Diafragma & 72 & $72 \%$ & 1 & $1 \%$ \\
\hline Espermicida & 58 & $58 \%$ & 2 & $2 \%$ \\
\hline DIU & 78 & $78 \%$ & 7 & $7 \%$ \\
\hline Temperatura Basal & 40 & $40 \%$ & 1 & $1 \%$ \\
\hline Billings “Muco cervical” & 41 & $41 \%$ & 1 & $1 \%$ \\
\hline Sintotérmico & 35 & $35 \%$ & 0 & $0 \%$ \\
\hline Vasectomia & 76 & $76 \%$ & 0 & $0 \%$ \\
\hline Laqueadura Tubária & 77 & $77 \%$ & 3 & $3 \%$ \\
\hline
\end{tabular}

\section{Discussão}

No presente estudo, observou-se, a partir dos dados coletados, que $100 \%$ das universitárias afirmaram conhecer algum tipo de método contraceptivo, 87\% asseguraram conhecer a camisinha feminina, sendo um fato interessante que somente $24 \%$ referem utilizá-la, A pesquisa não avaliou o motivo dessa ocorrência. No estudo de Cabral (2003), é salientado que as literaturas de um modo geral rela- 
tam que o principal obstáculo para o uso de algum método contraceptivo refere-se ao nível de conhecimento e informações disponíveis sobre eles. Figueiredo e Ayres (2002) realizaram um estudo-intervenção de base comunitária, buscando desenvolver e avaliar um conjunto de ações de prevenção das DSTs e da AIDS, dentre eles o uso da camisinha feminina, e destacou que, após a distribuição de material educativo, aulas e orientações, houve um interesse pelo uso da camisinha feminina. Esta pesquisa demonstrou que, das universitárias estudadas, o método contraceptivo mais utilizado (80\%) foi a camisinha masculina e que 58\% usaram contraceptivo na primeira relação. Na pesquisa de Faray (2008), esse fato também foi observado, assim como o uso predominante da camisinha masculina (condom).

Segundo Szwarcwald et al. (1985), mulheres jovens fazem maior uso de contraceptivos orais, principalmente aquelas de nível de instrução mais elevado, pois esse método tem menos chances de falhar. Esse comportamento foi observado em $68 \%$ das entrevistadas, que afirmaram usar a pílula anticoncepcional.

Nossa pesquisa mostrou que a idade em que as jovens iniciaram sua vida sexual foi entre 16 e 21 anos (60\%). Faray (2008) descreve em seu estudo que as relações sexuais têm sido iniciadas precocemente e também observou que grande parte das jovens universitárias respondeu ter iniciado sua vida sexual com idade entre 19 e 20 anos. Foi identificado que 11\% de universitárias se afirmaram ser "virgens".

O comportamento contraceptivo entre casadas e solteiras tem características diferentes. Nesta pesquisa, observou-se somente o conhecimento e comportamento em relação à prática e uso dos contraceptivos. As solteiras (80\%) confirmaram utilizar o preservativo masculino como método de anticoncepção seguido da pílula (75\%). Segundo Lima et al. (2006), no Rio de Janeiro a contracepção das adolescentes casadas é mais comum que entre as adultas.

Na pesquisa, foi perguntado quantos parceiros as universitárias haviam tido desde que iniciaram sua vida sexual ativa; a porcentagem de mulheres que afirmaram ter de 2 a 5 parceiros foi de $41 \%$, e $32 \%$ somente 1 parceiro.

Assim, percebe-se que as universitárias têm conhecimento considerável em relação à existência dos métodos contraceptivos, mas a prática delas precisa de novos esclarecimentos, já que, apesar de demonstrarem preocupação em evitar as 
doenças sexualmente transmissíveis, grande parte (80\%) alegou utilizar apenas a camisinha masculina.

A dúvida das universitárias, principalmente as do curso de Enfermagem, faz-se pela carência de estratégias educacionais direcionadas a esses grupos que serão multiplicadores e formadores de opiniões. As profissionais de Enfermagem são responsáveis por se familiarizarem com os vários métodos contraceptivos: seus benefícios e desvantagens, contraindicações, eficácia, segurança e custo.

\section{The knowledge of contraceptive methods among nursing academics}

\section{Abstract}

The sexual and reproductive behavior of the human being becomes related it the schooling level, therefore those one dedicate more to the studies make use of the contraceptive methods and prevent the undesirable pregnancy and/or the STDs. This research of quantitative boarding was possible from a questionnaire auto-applied on the study of transversal cut. The sample was composed for one hundred college students with average age 26 years old. The majority declared to have knowledge of some contraceptive method, but how about to the use, distortions had been identified. It was verified, that the knowledge affirmed between the college students seems not to establish coherent relation to the necessary auto-care for a planned and safe contraceptive behavior. It was verified, therefore, that better strategies guide these nursing academics who will go to compose the picture of the professionals of health in the future.

Keywords: Schooling. Behavior. Contraception.

\section{Referências}

BASTOS, M. R. et al. Práticas contraceptivas entre jovens universitárias: o uso da anticoncepção de emergência. Texto \& Contexto Enfermagem, Florianópolis, v. 17, n. 3, p. 447-455, jul./set. 2008.

CABRAL, C. S. Contracepção e gravidez na adolescência na perspectiva de jovens pais de uma comunidade favelada do Rio de Janeiro. Ciência e Saúde Coletiva, Rio de Janeiro, v. 19, p. S283-S292, 2003. Suplemento 2. 
COSTA, A. M. et al. Planejamento familiar: a autonomia das mulheres sob questão. Revista Brasileira de Saúde Materno Infantil, Recife, v. 6, n. 1, p. 75-84, 2006.

FARAY, H. H. F. G. Fatores determinantes da prática de métodos contraceptivos entre universitárias da área saúde. 2006. 46 f. Dissertação (Mestrado)-Saúde Materno-infantil. Universidade Federal do Maranhão, São Luis, 2006.

FIGUEIREDO, R.; AYRES, J. R. C. M. Intervenção comunitária e redução da vulnerabilidade de mulheres às DST/AIDS em São Paulo, SP. Revista de Saúde Pública, São Paulo, v. 36, n. 4, p. 96-107, ago. 2002. Suplemento.

LIMA, B. G. C. et al. Uso de contraceptivos e abortamento entre adolescentes. Revista Baiana de Saúde Pública, Salvador, v. 30, n. 2, p. 284-293, jul./dez. 2006.

LOPES, E. M. et al. Uso de métodos contraceptivos e incidência de gravidezes entre universitários da área de saúde. Disponível em: <www.enapet.ufsc.br/anais. php>. Acesso em: 22 out. 2009.

LOYOLA, M. A. Sexualidade e medicina: a revolução do século XX. Cadernos de Saúde Pública, Rio de Janeiro, v. 19, n. 4, p. 875884, jul./ago 2003.

MOURA, E. R. F.; SILVA, R. M. Competência profissional e assistência em anticoncepção. Revista de Saúde Pública, São Paulo, v. 39, n. 5, p. 795-801, out. 2005.

NETTINA, S. M. Métodos contraceptivos. In: NETTINA, S. M. Prática de enfermagem. 8.ed. Rio de Janeiro: Guanabara Koogan, 2007. cap. 22.

PIROTTA, K. C. M.; SCHOR, N. Intenções reprodutivas e práticas de regulação da fecundidade entre universitários. Revista de Saúde Pública, São Paulo, v. 38, n. 4, p. 495-502, jul./ago. 2004.

SANTOS, T. L. et al. Conhecimento e práticas contraceptivas entre as professoras universitárias. Revista Nursing, Aracaju, n. 12, p. 280-284, jun. 2009.

SMELTZER, S. C.; BARE, B. G. Contracepção. In: SMELTZER, S. C.; BARE, B. G. Brunner e Suddarth: tratado de enfermagem médico-cirúrgica. 10. ed. Rio de Janeiro: Guanabara Koogan, 2004. cap. 10.

SZWARCWALD, Célia L. et al. Anticoncepcionais orais e pressão arterial: pesquisa epidemiológica de hipertensão arterial no Rio Grande do Sul. Cadernos de Saúde Pública, Rio de Janeiro, v. 1, n. 2, p.177-191, abr./jun. 1985. 
Para publicar na revista Universitas Ciências da Saúde, entre no endereço eletrônico www.publicacoesacademicas.uniceub.br. Observe as normas de publicação, facilitando e agilizando o trabalho de edição. 